\title{
THERMOELASTIC STRONGLY NONLINEAR VIBRATION OF ROTATING FUNCTIONALLY GRADED RING PLATE
}

\author{
Yu Da Hu, Hai Jun Bao, Hao Ran Xu \\ School of Civil Engineering and Mechanics, Yanshan University, Qinhuangdao, China, and \\ Hebei Key Laboratory of Mechanical Reliability for Heavy Equipment and Large Structures, Yanshan University, \\ Qinhuangdao, China \\ e-mail: huyuda03@163.com (corresponding author: Yu Da Hu)
}

\begin{abstract}
For a metal ceramic functionally graded (FGM) ring plate, considering variations in physical properties with temperature and a power-law distribution of material components along the thickness direction, thermoelastic coupled nonlinear vibration equation in thermal environment is derived by means of Kirchhoff's thin plate theory and the Hamiltonian principle. The transverse nonlinear vibration differential equation of the inner and outside-clamped ring plate under static load is obtained by using the Galerkin method; moreover, perturbation solution of static deflection is carried out. An improved L-P method is employed to solve the strongly nonlinear vibration equation. The vibration response and nonlinear natural frequency expression are developed. Through numerical examples, natural frequency characteristic curves of the rotating FGM ring plate are plotted. The Runge-Kutta method is applied to obtain vibration response, phase and power spectrum diagrams. The influence of different parameters on natural vibration characteristics is analyzed. The results show that analytical solutions are consistent with numerical solutions, and the natural frequency decreases with an increase in the metal content and surface temperature, but grows with an increase in the rotational speed.
\end{abstract}

Keywords: functionally graded ring plate, natural vibration, rotational motion, strong nonlinearity, improved L-P method

\section{Introduction}

In practical engineering applications, FGM ring plates are widely used in space and rotating mechanical structures such as space shuttles engine combustion chambers, bearings, disks, etc. Most of them may exhibit complex dynamic behavior because of operation in complex coupled fields. Particularly, the existence of a temperature field affects stability and the running state of ring plate components. Since a metal ceramic FGM can reduce thermal deformation and relieve thermal stress at high temperature, a ring plate made of a metal ceramic FGM has a wide range of applications in many fields, so it is particularly important to study vibration of metal ceramic FGM ring plates.

Investigators have reported and discussed the dynamic behavior of rotating ring plate structures. Lamb and Southwell (1921) and Southwell (1992) were first to study the traveling wave dynamics of a rotating circular thin plate. Norouzi and Younesian (2015) studied forced vibration of a rotating disk under transverse load and obtained natural frequencies at different rotational speeds. Chen and Chen (2007) analyzed non-axisymmetric vibration and stability of a rotating sandwich ring plate by the finite element method and discussed the influence of variation of parameters such as stiffness and thickness on vibration of the system. Hashemi et al. (2009) combined the Mindlin plate theory with the second-order strain displacement hypothesis 
to establish a finite element formula for vibration analysis of rotating thick plates and studied linear and nonlinear differences of the in-plane vibration of rotating plates.

A lot of research has been done on dynamic problems of functionally graded structures (FGS). Hu et al. (2017) used the Bessel function as a mode function to study bifurcation and chaos of a rotating annular thin plate with different boundary conditions in a magnetic field. In addition, bending and vibration problems of FGM ring plates and shells in thermal environment were studied. Ma and Wang (2003, 2004) examined nonlinear bending and post buckling behavior of FGM circular plates under thermal mechanical loading. Yousefitabar and Matapouri (2017) analyzed stability of FGM annular plates under three different temperature loads and proposed an analytical solution for the thermal buckling load. Hu and Zhang (2012) discussed bifurcation and chaos of an FGM circular plate with a fixed support. Nosier and Fallah (2009) generalized the first-order shear deformation theory of von Karman and analyzed nonlinear axisymmetric and asymmetric behavior of FGM ring circular plates under transverse mechanical loads. Malekzadeh et al. (2011) used the difference orthogonal method to study free vibration of annular plates with thin and medium thickness functionally graded structure in thermal environment on the basis of the two-parameter elasticity. Żur (2018), based on the classical elastic theory, studied the problem of axisymmetric and non-axisymmetric free vibration of a gradient annular plate with an elastic support function. Dai and Dai (2016) obtained displacement and stress fields of an FGM hollow disk under a change in the temperature field by a semi-analytical method. Shakouri (2019) reported and analyzed free vibration of a functionally graded rotating conical shell in thermal environment.

At present, the research on FGS is mainly focused on the critical internal force, thermal stress and bending, however, the investigations on nonlinear vibration of FGS under coupled fields are few. In this paper, considering the rotation effect, thermoelastic coupled strongly nonlinear vibration of an FGM ring plate is investigated. Through analytical and numerical solutions, the free vibration response and the natural vibration frequency expression of the nonlinear system are obtained. In addition, the effect of the volume fraction index, rotational speed and temperature on vibration characteristics of the system are given.

\section{Basic equations}

Consider that the material of a ring plate in a temperature field from the upper surface to the lower surface is a gradually changing circular gradient from a metal to ceramics. As shown in Fig. 1, the inner diameter of the ring plate is $a$, the outer diameter is $b$, the plate thickness is $h$. The plate is rotating at the constant angular velocity $\Omega$.

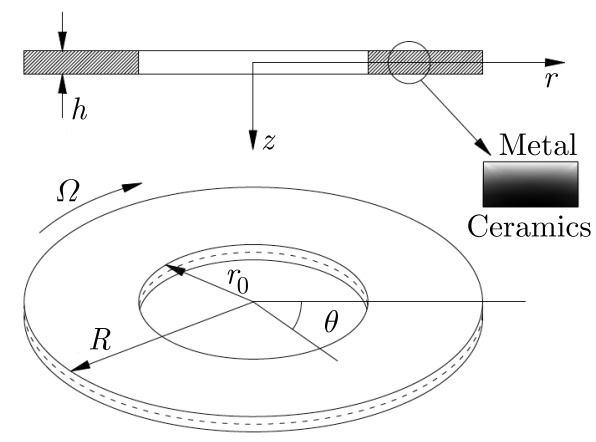

Fig. 1. The mechanical model of a rotating FGM ring plate 


\subsection{FGM property parameters}

The FGM properties present gradient changes along the thickness direction, and the material physical parameters are

$$
P=P_{c} V_{c}+P_{m} V_{m}=\left(P_{c}-P_{m}\right) V_{c}+P_{m}
$$

where $P_{c}$ and $P_{m}$ are physical parameters of ceramics and metal materials changing with temperature. $V_{c}$ and $V_{m}$ are volume fractions of the ceramics and metal, respectively

$$
\begin{aligned}
& P_{c, m}=P_{0}\left(P_{-1} T^{-1}+1+P_{1} T^{1}+P_{2} T^{2}+P_{3} T^{3}\right) \\
& V_{c}=\left(\frac{2 z+h}{2 h}\right)^{n} \quad V_{c}+V_{m}=1
\end{aligned}
$$

where $n$ is the volume fraction index; $P_{0}, P_{-1}, P_{1}, P_{2}$ and $P_{3}$ are temperature correlation coefficients determined by the material properties and experimental results.

It is assumed that Young's modulus $E$ and thermal expansion coefficient $\alpha$ are temperaturedependent, the heat conductivity $\kappa$ and density $\rho$ are independent of temperature, and Poisson's ratio $\nu$ is set as a constant (Shen, 2007)

$$
\begin{aligned}
& E(z, T)=\left[E_{c}(T)-E_{m}(T)\right]\left(\frac{2 z+h}{2 h}\right)^{n}+E_{m}(T) \\
& \alpha(z, T)=\left[\alpha_{c}(T)-\alpha_{m}(T)\right]\left(\frac{2 z+h}{2 h}\right)^{n}+\alpha_{m}(T) \\
& \kappa(z)=\left(\kappa_{c}-\kappa_{m}\right)\left(\frac{2 z+h}{2 h}\right)^{n}+\kappa_{m} \quad \rho(z)=\left(\rho_{c}-\rho_{m}\right)\left(\frac{2 z+h}{2 h}\right)^{n}+\rho_{m}
\end{aligned}
$$

where $E_{c}$ and $E_{m}$ are Young's modulus, $\alpha_{c}$ and $\alpha_{m}$ are temperature-dependent expansion coefficients, $\kappa_{c}$ and $\kappa_{m}$ are heat conductivities, $\rho_{c}$ and $\rho_{m}$ are densities of the ceramic and metal materials, respectively.

\subsection{Heat conduction}

According to the treatment method in (Yousefitabar and Matapouri, 2017; Hu and Zhang, 2012; Shen, 2007), the temperature distribution function is determined by a steady-state heat conduction equation and the heat boundary condition. It is assumed that the temperature field of the FGM ring plate is uniform in the plane of $r \theta$. The temperature field varies nonuniformly along the thickness, but does not change with time and structural deformation. The one-dimensional heat conduction equation is as follows

$$
-\frac{d}{d z}\left[\kappa(z) \frac{d T}{d z}\right]=0
$$

The thermal boundary conditions are

$$
T\left(\frac{h}{2}\right)=T_{c} \quad T\left(-\frac{h}{2}\right)=T_{m}
$$

The solution of Eq. (2.4) can be expressed by the following polynomials (Shen, 2007)

$$
\Delta T(z)=\Delta T_{m}+\left(\Delta T_{c}-\Delta T_{m}\right) \eta(z)
$$


where $\Delta T_{m}$ and $\Delta T_{c}$ represent the temperature rise value from the initial temperature, and

$$
\begin{aligned}
\eta(z) & =\frac{1}{\beta}\left[\left(\frac{2 z+h}{2 h}\right)-\frac{\kappa_{c m}}{(n+1) \kappa_{m}}\left(\frac{2 z+h}{2 h}\right)^{n+1}+\frac{\kappa_{c m}^{2}}{(2 n+1) \kappa_{m}^{2}}\left(\frac{2 z+h}{2 h}\right)^{2 n+1}\right. \\
& \left.-\frac{\kappa_{c m}^{3}}{(3 n+1) \kappa_{m}^{3}}\left(\frac{2 z+h}{2 h}\right)^{3 n+1}+\frac{\kappa_{c m}^{4}}{(4 n+1) \kappa_{m}^{4}}\left(\frac{2 z+h}{2 h}\right)^{4 n+1}-\frac{\kappa_{c m}^{5}}{(5 n+1) \kappa_{m}^{5}}\left(\frac{2 z+h}{2 h}\right)^{5 n+1}\right] \\
\beta= & 1-\frac{\kappa_{c m}}{(n+1) \kappa_{m}}+\frac{\kappa_{c m}^{2}}{(2 n+1) \kappa_{m}^{2}}-\frac{\kappa_{c m}^{3}}{(3 n+1) \kappa_{m}^{3}}+\frac{\kappa_{c m}^{4}}{(4 n+1) \kappa_{m}^{4}}-\frac{\kappa_{c m}^{5}}{(5 n+1) \kappa_{m}^{5}} \\
\kappa_{c m} & =\kappa_{c}-\kappa_{m}
\end{aligned}
$$

Then, the thermal internal force can be expressed as

$$
N_{T}=\int_{-h / 2}^{h / 2} \frac{E(z, T)}{1-\nu}[\alpha(z, T) \Delta T(z)] d z \quad M_{T}=\int_{-h / 2}^{h / 2} \frac{z E(z, T)}{1-\nu}[\alpha(z, T) \Delta T(z)] d z
$$

\subsection{Kinetic energy and deformation potential energy}

Let the deformation displacement components of any point in the ring plate be

$$
\begin{aligned}
& u_{r 1}(r, \theta, z, t)=u_{r}(r, \theta, t)-z \frac{\partial w}{\partial r} \quad u_{\theta 1}(r, \theta, z, t)=u_{\theta}(r, \theta, t)-\frac{z}{r} \frac{\partial w}{\partial \theta} \\
& w_{1}(r, \theta, z, t)=w(r, \theta, t)
\end{aligned}
$$

where $u_{r}, u_{\theta}$ and $w$ are the midplane and transverse displacement of the ring plate, respectively; $t$ is the time variable.

Considering the effect of rotation, the expression for kinetic energy in rotating motion is given as

$$
T=\frac{1}{2} \int_{a}^{b} \int_{0}^{2 \pi} \int_{-h / 2}^{h / 2} \rho(z)\left[\left(\frac{d u_{r 1}}{d t}-\Omega u_{\theta 1}\right)^{2}+\left(\frac{d u_{\theta 1}}{d t}+\Omega\left(u_{r 1}+r\right)\right)^{2}+\left(\frac{d w}{d t}\right)^{2}\right] d r d \theta d z
$$

According to the classical Kirchhoff plate theory, considering geometric nonlinearity and axisymmetry, the expressions for the internal force and bending moment in the middle plane of a ring plate are obtained as follows

$$
\begin{aligned}
N_{r}=\int_{-h / 2}^{h / 2} \frac{E(z, T)}{1-\nu^{2}}\left(\varepsilon_{r}+\nu \varepsilon_{\theta}\right) d z-N_{T} & N_{\theta}=\int_{-h / 2}^{h / 2} \frac{E(z, T)}{1-\nu^{2}}\left(\nu \varepsilon_{r}+\varepsilon_{\theta}\right) d z-N_{T} \\
M_{r}=\int_{-h / 2}^{h / 2} \frac{z E(z, T)}{1-\nu^{2}}\left(\varepsilon_{r}+\nu \varepsilon_{\theta}\right) d z-M_{T} & M_{\theta}=\int_{-h / 2}^{h / 2} \frac{z E(z, T)}{1-\nu^{2}}\left(\nu \varepsilon_{r}+\varepsilon_{\theta}\right) d z-M_{T}
\end{aligned}
$$

where

$$
\varepsilon_{r}=\frac{\partial u_{r}}{\partial r}+\frac{1}{2}\left(\frac{\partial w}{\partial r}\right)^{2}-z \frac{\partial^{2} w}{\partial r^{2}} \quad \varepsilon_{\theta}=\frac{u_{r}}{r}-\frac{z}{r} \frac{\partial w}{\partial r}
$$

are strain components in the middle plane. 
For axisymmetric problems, the potential energy $U_{\varepsilon 1}$ of bending deformation and potential energy $U_{\varepsilon 2}$ of middle plane strain caused by deformation of the rotating ring plate are expressed as

$$
U_{\varepsilon 1}=\frac{1}{2} \int_{0}^{R} \int_{0}^{2 \pi}\left(M_{r} k_{r}+M_{\theta} k_{\theta}\right) r d r d \theta \quad U_{\varepsilon 2}=\frac{1}{2} \int_{0}^{R} \int_{0}^{2 \pi}\left(N_{r} \varepsilon_{r}+N_{\theta} \varepsilon_{\theta}\right) r d r d \theta
$$

where $k_{r}$ and $k_{\theta}$ are the middle plane curvatures.

\subsection{Nonlinear vibration equation}

According to the Hamiltonian variational principle, we can get

$$
\int_{t_{1}}^{t_{2}}\left(\delta T-\delta U_{\varepsilon 1}-\delta U_{\varepsilon 2}\right) d t=0
$$

where $t_{1}$ and $t_{2}$ describe the fixed integral time domain of integration.

Substituting Eqs. (2.10) to (2.12) into Eq. (2.13) and performing variational operations on the displacement components $u_{r}, u_{\theta}$ and $w$, the integral expressions for $\delta u_{r}, \delta u_{\theta}$ and $\delta w$ as independent variable components can be obtained. To satisfy Eq. (2.13), the terms corresponding to $\delta u_{r}, \delta u_{\theta}$ and $\delta w$ in the $r, \theta$ and $z$ directions are zero. Here, the axisymmetric vibration of the plate is studied. Only vibrations in the direction of $r$ and $z$ are considered, hence, only the two expressions of $\delta u_{r}$ and $\delta w$ are zero. Then, we obtain the vibration equations

$$
\begin{aligned}
- & B_{1}\left(\frac{\partial^{2} u_{r}}{\partial r^{2}}+\frac{1}{r} \frac{\partial u_{r}}{\partial r}-\frac{1}{r^{2}} u_{r}\right)-B_{1} \frac{1-\nu}{2 r}\left(\frac{\partial w}{\partial r}\right)^{2}-B_{1} \frac{\partial w}{\partial r} \frac{\partial^{2} w}{\partial r^{2}} \\
+ & B_{2}\left(\frac{\partial^{3} w}{\partial r^{3}}+\frac{1}{r} \frac{\partial^{2} w}{\partial r^{2}}-\frac{1}{r^{2}} \frac{\partial w}{\partial r}\right)+A_{1} \frac{\partial^{2} u_{r}}{\partial t^{2}}-A_{1} \Omega^{2}\left(r+u_{r}\right)+A_{2} \Omega^{2} \frac{\partial w}{\partial r}=0 \\
- & B_{1}\left(\nabla^{2} u_{r}+\frac{\nu}{r} \frac{\partial u_{r}}{\partial r}\right) \frac{\partial w}{\partial r}-B_{1}\left(\frac{\partial u_{r}}{\partial r}+\frac{\nu}{r} u_{r}\right) \frac{\partial^{2} w}{\partial r^{2}}-B_{1}\left[\frac{1}{2 r}\left(\frac{\partial w}{\partial r}\right)^{3}+\frac{3}{2}\left(\frac{\partial w}{\partial r}\right)^{2} \frac{\partial^{2} w}{\partial r^{2}}\right] \\
+ & B_{3} \nabla^{4} w+N_{T} \nabla^{2} w-B_{2}\left[\frac{\partial^{3} u_{r}}{\partial r^{3}}+\frac{2}{r} \frac{\partial^{2} u_{r}}{\partial r^{2}}-\frac{1}{r^{2}} \frac{\partial u_{r}}{\partial r}+\frac{1}{r^{3}} u_{r}\right]+A_{1} \frac{\partial^{2} w}{\partial t^{2}} \\
- & A_{3}\left(\frac{\partial^{4} w}{\partial r^{2} \partial t^{2}}+\frac{1}{r} \frac{\partial^{3} w}{\partial r \partial t^{2}}\right)-B_{2}\left[\frac{1-3 \nu)}{r} \frac{\partial w}{\partial r} \frac{\partial^{2} w}{\partial r^{2}}\right]+A_{3} \Omega^{2} \nabla^{2} w \\
- & A_{2} \Omega^{2}\left(\frac{\partial u_{r}}{\partial r}+\frac{1}{r} u_{r}+2\right)=0
\end{aligned}
$$

where $\nabla^{2}=\left(\partial^{2} / \partial r^{2}\right)+(\partial / \partial r) / r$ is the differential operator. $B_{1,2,3}$ and $A_{1,2,3}$ are coefficients of stiffness

$$
B_{1,2,3}=\int_{-h / 2}^{h / 2} \frac{E(z, T)}{1-\nu^{2}}\left(1, z, z^{2}\right) d z \quad A_{1,2,3}=\int_{-h / 2}^{h / 2} \rho(z)\left(1, z, z^{2}\right) d z
$$

\section{Galerkin discretization and static load effect}

This paper studies a ring plate with the inner edge fixed and clamped, and the outer edge constrained by clamped boundary constraints. The boundary conditions are

$$
\begin{aligned}
& \left.u_{r}\right|_{r=a}=\left.0 \quad w\right|_{r=a}=\left.0 \quad \frac{\partial w}{\partial r}\right|_{r=a}=0 \\
& \left.w\right|_{r=b}=\left.0 \quad \frac{\partial w}{\partial r}\right|_{r=b}=\left.0 \quad\left(\nu \frac{\partial u_{r}}{\partial r}+\frac{1}{r} u_{r}\right)\right|_{r=b}=0
\end{aligned}
$$


The time and space variables are separated. According to formulation of the displacement function (Li et al., 2011; Chonan et al., 1986), the displacement solution in the $r$ and $z$ directions satisfying the boundary condition is set as follows

$$
u_{r}=f U \quad w=g(t) W
$$

where

$$
U=\left(\frac{r}{b}-\frac{a}{b}\right)\left(\frac{r}{b}-d\right) \quad W=1+\frac{r}{b}+\frac{r^{4}}{b^{4}}+E_{1} \frac{r^{2}}{b^{2}}+E_{2} \frac{r^{3}}{b^{3}}+E_{3} \frac{r^{5}}{b^{5}}+E_{4} \frac{r^{6}}{b^{6}}
$$

Substituting Eq. (3.2) into Eq. (3.1), in order to meet the boundary conditions, the coefficients in the displacement function can be obtained as follows

$$
\begin{aligned}
& d=1+\frac{b-a}{b+\nu(b-a)} \quad E_{1}=-\frac{4 a^{4}+20 a^{3} b+23 a^{2} b^{2}+14 a b^{3}+3 b^{4}}{a^{2}\left(a^{2}+4 a b+b^{2}\right)} \\
& E_{2}=\frac{a^{5}+10 a^{4} b+30 a^{3} b^{2}+24 a^{2} b^{3}+9 a b^{4}+2 b^{5}}{a^{3}\left(a^{2}+4 a b+b^{2}\right)} \\
& E_{3}=-\frac{b\left(2 a^{4}+11 a^{3} b+16 a^{2} b^{2}+15 a b^{3}+6 b^{4}\right)}{a^{3}\left(a^{2}+4 a b+b^{2}\right)} \\
& E_{4}=\frac{b^{2}\left(a^{3} b+6 a^{2} b+9 a b^{2}+4 b^{3}\right)}{a^{3}\left(a^{2}+4 a b+b^{2}\right)}
\end{aligned}
$$

Substituting the displacement function in Eq. (3.2) into Eqs. (2.14), yields

$$
\begin{aligned}
& a_{1} f+a_{2} g^{2}+a_{3} g+a_{4}=0 \\
& b_{1} \ddot{g}+b_{2} g+b_{3} g^{2}+b_{4} g^{3}+b_{5} f+b_{6} f g+b_{7}=0
\end{aligned}
$$

where

$$
\begin{aligned}
& a_{1}=\int_{a}^{b}\left[-B_{1}\left(U^{\prime \prime}+\frac{1}{r} U^{\prime}-\frac{1}{r^{2}} U\right)-A_{1} \Omega^{2} U\right] U r d r \\
& a_{2}=\int_{a}^{b}\left[-B_{1}\left(W^{\prime} W^{\prime \prime}+\frac{1-\nu}{2 r}\left(W^{\prime}\right)^{2}\right)\right] U r d r \\
& a_{3}=\int_{a}^{b}\left[B_{2}\left(W^{\prime \prime \prime}+\frac{1}{r} W^{\prime \prime}-\frac{1}{r^{2}} W^{\prime}\right)+A_{2} \Omega^{2} W^{\prime}\right] U r d r \quad a_{4}=-\int_{a}^{b} A_{1} \Omega^{2} U r^{2} d r \\
& b_{1}=\int_{a}^{b}\left[A_{1} W-A_{3}\left(W^{\prime \prime}+\frac{1}{r} W^{\prime}\right)\right] W r d r \\
& b_{2}=\int_{a}^{b}\left[B_{3}\left(W^{i v}+\frac{2}{r} W^{\prime \prime \prime}-\frac{1}{r^{2}} W^{\prime \prime}+\frac{1}{r^{3}} W^{\prime}\right)+\left(N_{T}+A_{3} \Omega^{2}\right)\left(W^{\prime \prime}+\frac{1}{r} W^{\prime}\right)\right] W r d r \\
& b_{3}=-\int_{a}^{b}\left[B_{2} \frac{1-3 \nu}{r} W^{\prime \prime} W^{\prime}\right] W r d r \quad b_{a}=-B_{1}\left[\frac{1}{2 r}\left(W^{\prime}\right)^{3}+\frac{3}{2}\left(W^{\prime}\right)^{2}\left(W^{\prime \prime}\right)\right] W r d r \\
& b_{5}=-\int_{a}^{b}\left[B_{2}\left(U^{\prime \prime \prime}+\frac{2}{r} U^{\prime \prime}-\frac{1}{r^{2}} U^{\prime}+\frac{1}{r^{3}} U\right)+A_{2} \Omega^{2}\left(U^{\prime \prime}+\frac{1}{r} U^{\prime}\right)\right] W r d r \\
& b_{6}=-\int_{a}^{b} B_{1}\left[\left(U^{\prime \prime}+\frac{1+\nu}{r} U^{\prime}\right) W^{\prime}+\left(U^{\prime}+\frac{\nu}{r} U\right) W^{\prime \prime}\right] W r d r \quad b_{7}=-\int_{a}^{b} 2 A_{2} \Omega^{2} W r d r
\end{aligned}
$$


According to Eqs. (3.4), the transverse vibration equation of the ring plate can be obtained

$$
d_{1} \ddot{g}+d_{2} g+d_{3} g^{2}+d_{4} g^{3}+d_{5}=0
$$

where

$$
\begin{aligned}
& d_{1}=b_{1} \quad d_{2}=b_{2}-\frac{a_{3} b_{5}}{a_{1}}-\frac{a_{4} b_{6}}{a_{1}} \quad d_{3}=b_{3}-\frac{a_{2} b_{5}}{a_{1}}-\frac{a_{3} b_{6}}{a_{1}} \\
& d_{4}=b_{4}-\frac{a_{2} b_{6}}{a_{1}} \quad d_{5}=b_{7}-\frac{a_{4} b_{5}}{a_{1}}
\end{aligned}
$$

It can be seen from Eq. (3.5) that during rotation of the ring plate, the static deflection $g_{0}$ caused by the static load term is generated in the direction of $z$. Then, the deflection of the ring plate can be expressed as

$$
g(t)=g_{0}+g_{1}(t)
$$

where $g_{1}(t)$ is the disturbance deflection, and the static deflection $g_{0}$ should meet the following requirement

$$
d_{2} g_{0}+d_{3} g_{0}^{2}+d_{4} g_{0}^{3}+d_{5}=0
$$

The solution of Eq. (3.7) can be expressed as

$$
g_{0}=-\frac{d_{4}}{3 d_{5}}+\sqrt[3]{-e_{1}+\sqrt{e_{1}^{2}+e_{2}^{3}}}+\sqrt[3]{-e_{1}-\sqrt{e_{1}^{2}+e_{2}^{3}}}
$$

where

$$
e_{1}=\frac{27 d_{4}^{2} d_{5}-9 d_{2} d_{3} d_{4}+2 d_{3}^{3}}{54 d_{4}^{3}} \quad e_{2}=\frac{3 d_{2} d_{4}-d_{3}^{2}}{9 d_{4}^{2}}
$$

Finally, by substituting Eq. (3.6) into Eq. (3.5) and considering Eq. (3.8), the nonlinear perturbation differential equation of the ring plate can be derived

$$
\ddot{x}(t)+\omega_{0}^{2} x(t)+\widetilde{\eta}_{1} x^{2}(t)+\widetilde{\eta}_{2} x^{3}(t)=0
$$

where

$$
x=g_{1} \quad \widetilde{\eta}_{1}=\frac{d_{3}+3 d_{4} g_{0}}{d_{1}} \quad \widetilde{\eta}_{2}=\frac{d_{4}}{d_{1}} \quad \omega_{0}^{2}=\frac{d_{2}+2 d_{3} g_{0}+3 d_{4} g_{0}^{2}}{d_{1}}
$$

\section{Improved L-P method for solving strongly nonlinear vibration equation}

Considering that the differential equation of the FGM ring plate in thermal environment is strongly non-linear, $\varepsilon$ is introduced into Eq. (3.9), $\varepsilon>0 . \varepsilon$ is not a small parameter, and with $\eta_{1}=\widetilde{\eta}_{1} / \varepsilon, \eta_{2}=\widetilde{\eta}_{2} / \varepsilon$, one gets

$$
\ddot{x}(t)+\omega_{0}^{2} x(t)+\varepsilon \eta_{1} x^{2}(t)+\varepsilon \eta_{2} x^{3}(t)=0
$$

For convenience of solution and analysis, the time variables are transformed as follows

$$
\omega^{2} \ddot{x}(\tau)+\omega_{0}^{2} x(\tau)+\varepsilon \eta_{1} x^{2}(\tau)+\varepsilon \eta_{2} x^{3}(\tau)=0
$$

where $\tau=\omega t, \omega$ is the nonlinear natural vibration frequency of the original system to be determined. 
To solve nonlinear Eq. (4.2), the transformation parameters are introduced

$$
\lambda=\frac{\varepsilon \omega_{1}}{\omega_{0}^{2}+\varepsilon \omega_{1}}
$$

Further, $\omega^{2}$ is expanded into a power series in $\lambda$ near $\omega_{0}^{2}$, which gives

$$
\omega^{2}=\frac{\omega_{0}^{2}}{1-\lambda}\left(1+\delta_{2} \lambda^{2}+\delta_{3} \lambda^{3}+\cdots\right)
$$

where $\omega_{1}$ and $\delta_{i}(i=1,2,3, \ldots)$ are undetermined transformation constants.

Expanding $x$ in Eq. (4.2) into a power series in the new parameter $\lambda$, one obtains

$$
x=\sum_{n=0}^{\infty} \lambda^{n} x_{n}=x_{0}+\lambda x_{1}+\lambda^{2} x_{2}+\cdots
$$

Substituting Eqs. (4.3), (4.4) and (4.5) into Eq. (4.2) and collecting the coefficients of the zeroth and first power terms of $\lambda$, we get approximate linear partial differential equations in each order as

$$
\begin{aligned}
& \ddot{x}_{0}+x_{0}=0 \\
& \ddot{x}_{1}+x_{1}=x_{0}-\frac{\eta_{1}}{\omega_{1}} x_{0}^{2}-\frac{\eta_{2}}{\omega_{1}} x_{0}^{3} \\
& \ddot{x}_{2}+x_{2}=x_{1}-\delta_{2} \ddot{x}_{0}-\frac{2 \eta_{1}}{\omega_{1}} x_{0} x_{1}-\frac{3 \eta_{2}}{\omega_{1}} x_{0}^{2} x_{1}
\end{aligned}
$$

The initial values are $x_{0}(0)=a_{0}, \dot{x}_{0}(0)=0$. Let the solution satisfying Eq. $(4.6)_{1}$ be

$$
x_{0}=a_{0} \cos \tau
$$

Substituting Eq. (4.7) into formula Eq. (4.6) $)_{2}$, yields

$$
\ddot{x}_{1}+x_{1}=\left(a_{0}-\frac{3 \eta a_{0}^{3}}{4 \omega_{1}}\right) \cos \tau-\frac{\eta_{1} a_{0}^{2}}{2 \omega_{1}} \cos 2 \tau-\frac{\eta_{2} a_{0}^{3}}{4 \omega_{1}} \cos 3 \tau-\frac{\eta_{1} a_{0}^{2}}{2 \omega_{1}}
$$

To eliminate the duration item, we obtain

$$
\omega_{1}=\frac{3 \eta_{2} a_{0}^{2}}{4}
$$

The solution of Eq. (4.8) is

$$
x_{1}=\frac{\eta_{1} a_{0}^{2}}{6 \omega_{1}} \cos 2 \tau+\frac{\eta_{2} a_{0}^{3}}{32 \omega_{1}} \cos 3 \tau-\frac{\eta_{1} a_{0}^{2}}{2 \omega_{1}}
$$

Similarly, substituting Eqs. (4.7) and (4.10) into Eq. (4.6) $)_{3}$ and eliminating the duration term, we obtain

$$
\delta_{2}=\frac{3 \eta_{2}^{2} a_{0}^{4}}{128 \omega_{1}^{2}}-\frac{5 \eta_{1}^{2} a_{0}^{2}}{6 \omega_{1}^{2}}
$$

and

$$
\begin{aligned}
x_{2}= & -\left(\frac{5 \eta_{1} \eta_{2} a_{0}^{4}}{32 \omega_{1}^{2}}+\frac{\eta_{1} a_{0}^{2}}{18 \omega_{1}}\right) \cos 2 \tau-\left(\frac{\eta_{2} a_{0}^{3}}{256 \omega_{1}}-\frac{\eta_{1}^{2} a_{0}^{5}}{48 \omega_{1}^{2}}-\frac{3 \eta_{2}^{2} a_{0}^{3}}{256 \omega_{1}}\right) \cos 3 \tau \\
& +\frac{\eta_{1} \eta_{2} a_{0}^{4}}{96 \omega_{1}^{2}} \cos 4 \tau+\frac{\eta_{2}^{2} a_{0}^{5}}{1024 \omega_{1}^{2}} \cos 5 \tau-\frac{\eta_{1} a_{0}^{2}}{2 \omega_{1}}+\frac{5 \eta_{1} \eta_{2} a_{0}^{4}}{8 \omega_{1}^{2}}
\end{aligned}
$$


Finally, substituting Eqs. (4.7), (4.10) and (4.12) into Eq. (4.5), the analytical solution is obtained as follows

$$
\begin{aligned}
x= & a_{0} \cos \tau+\left(\frac{\lambda \eta_{1} a_{0}^{2}}{6 \omega_{1}}-\frac{\lambda^{2} \eta_{1} a_{0}^{2}}{18 \omega_{1}}-\frac{5 \lambda^{2} \eta_{1} \eta_{2} a_{0}^{4}}{32 \omega_{1}^{2}}\right) \cos 2 \tau \\
& +\left(\frac{\lambda \eta_{2} a_{0}^{3}}{32 \omega_{1}}-\frac{\lambda^{2} \eta_{2} a_{0}^{3}}{256 \omega_{1}}+\frac{\lambda^{2} \eta_{1}^{2} a_{0}^{3}}{48 \omega_{1}^{2}}+\frac{3 \lambda^{2} \eta_{2}^{2} a_{0}^{5}}{512 \omega_{1}^{2}}\right) \cos 3 \tau+\frac{\lambda^{2} \eta_{1} \eta_{2} a_{0}^{4}}{96 \omega_{1}^{2}} \cos 4 \tau \\
& +\frac{\lambda^{2} \eta_{2}^{2} a_{0}^{5}}{1024 \omega_{1}^{2}} \cos 5 \tau-\frac{\lambda \eta_{1} a_{0}^{2}}{2 \omega_{1}}-\frac{\lambda^{2} \eta_{1} a_{0}^{2}}{2 \omega_{1}}+\frac{5 \lambda^{2} \eta_{1} \eta_{2} a_{0}^{4}}{8 \omega_{1}^{2}}
\end{aligned}
$$

It can be seen from Eq. (4.13) that the free vibration solution of the system contains five simple harmonic excitation and constant terms. The first-order nonlinear natural vibration frequency of the ring plate is

$$
\omega=\omega_{0}\left[1+\frac{1}{2} \lambda+\left(\frac{19}{48}-\frac{20 \widetilde{\eta}_{1}^{2}}{27 \widetilde{\eta}_{2}^{2} a_{0}^{2}}\right) \lambda^{2}+\cdots\right]
$$

where $\lambda=3 \widetilde{\eta}_{2} a_{0}^{2} /\left(4 \omega_{0}^{2}+3 \widetilde{\eta}_{2} a_{0}^{2}\right)$.

\section{Numerical results and discussions}

In this Section, vibration characteristics of the FGM ring plate with inner-clamped and the outer-clamped boundary conditions, rotating in thermal environment are analyzed. Selected is the inner diameter of the ring plate $a=0.3 \mathrm{~m}$, outer diameter $b=0.6 \mathrm{~m}$ and the initial surface temperature $T_{0}=300 \mathrm{~K}$. The FGM plate is composed of ceramics and metals. Density and thermal conductivity of the component materials are respectively: $\mathrm{Si}_{3} \mathrm{~N}_{4}, \rho_{c}=2370 \mathrm{~kg} / \mathrm{m}^{3}$, $\kappa_{c}=9.16 \mathrm{~W} /(\mathrm{mK}) ;$ SUS304, $\rho_{m}=8166 \mathrm{~kg} / \mathrm{m}^{3}, \kappa_{m}=12.04 \mathrm{~W} /(\mathrm{mK})$. The modulus of elasticity and coefficient of thermal expansion change with temperature. According to (Reddy and Chin, 1998), specific material data for physical parameters of metals and ceramics is listed in Table 1. Poisson's ratio is set constant $\nu=0.3$.

Table 1. Temperature-dependent coefficients for ceramics $\left(\mathrm{Si}_{3} \mathrm{~N}_{4}\right)$ and metals (SUS304)

\begin{tabular}{|c|c|c|c|c|c|c|}
\hline Material & $\begin{array}{c}\text { Physical } \\
\text { parameters }\end{array}$ & $P_{-1}$ & $P_{0}$ & $P_{1}$ & $P_{2}$ & $P_{3}$ \\
\hline \hline \multirow{2}{*}{$\mathrm{Si}_{3} \mathrm{~N}_{4}$} & $\alpha[1 / \mathrm{K}]$ & 0 & $5.8723 \mathrm{e}-6$ & $9.095 \mathrm{e}-4$ & 0 & 0 \\
\cline { 2 - 7 } & $E[\mathrm{~Pa}]$ & 0 & $348.43 \mathrm{e}+9$ & $-3.07 \mathrm{e}-4$ & $2.16 \mathrm{e}-7$ & $-8.946 \mathrm{e}-11$ \\
\hline \multirow{2}{*}{$\mathrm{SUS} 304$} & $\alpha[1 / \mathrm{K}]$ & 0 & $12.33 \mathrm{e}-6$ & $8.086 \mathrm{e}-4$ & 0 & 0 \\
\cline { 2 - 7 } & $E[\mathrm{~Pa}]$ & 0 & $201.04 \mathrm{e}+9$ & $3.079 \mathrm{e}-4$ & $-6.534 \mathrm{e}-7$ & 0 \\
\hline
\end{tabular}

\subsection{Law of change in natural frequency with different parameters}

Figure 2 shows characteristic curves of the first-order nonlinear natural vibration frequency of the rotating FGM ring plate with a change in the volume fraction index. Figure 2 presents that when the volume fraction index is from $n=0$ to $n=2$, the natural frequency drops rapidly, and then the rate of decrease gradually slows down. As the volume content of ceramics and metals and $n$ are power exponents, the metal content increases with $n$. Figure $2 \mathrm{a}(\Omega=3000 \mathrm{r} / \mathrm{min}$, $h=0.006 \mathrm{~m}, T_{c}=350 \mathrm{~K}$ and $T_{m}=300 \mathrm{~K}$ ) shows that the larger the initial amplitude, the greater the nonlinear natural frequency. In a linear system, the natural frequency is not dependent on the initial condition but only related to natural parameters of the system. In a nonlinear system, the 
frequency is affected by the initial amplitude. Figure $2 \mathrm{~b}\left(a_{0}=0.006 \mathrm{~m}, h=0.006 \mathrm{~m}, T_{c}=350 \mathrm{~K}\right.$ and $T_{m}=300 \mathrm{~K}$ ) shows that at the same volume fraction, the greater the speed, the greater the frequency. Figure 2c $\left(a_{0}=0.006 \mathrm{~m}, \Omega=3000 \mathrm{r} / \mathrm{min}, h=0.006 \mathrm{~m}\right)$ shows that as $n$ increases, the plate surface temperature affects the natural frequency. When only surface temperature of ceramics increases, the natural frequency decreases, but when temperature of both ceramic and metal surfaces increase, the frequency is smaller than that when only temperature on the ceramic side increases.

(a)

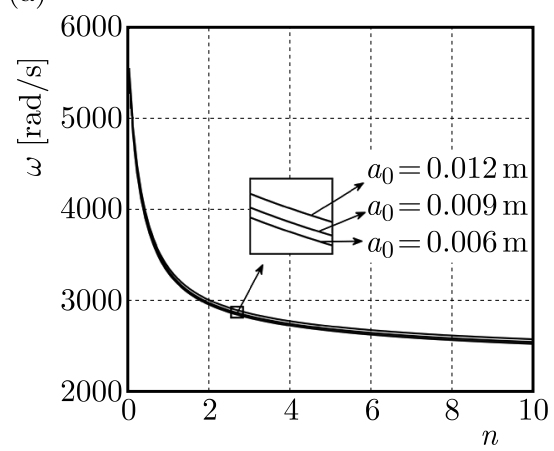

(b)

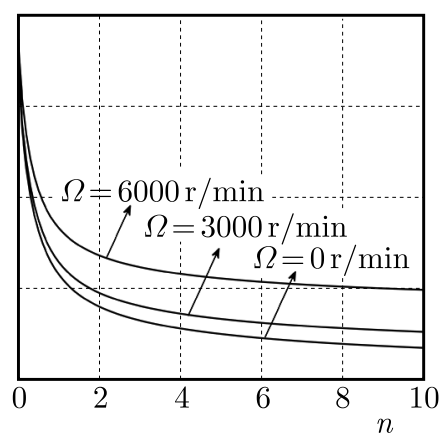

(c)

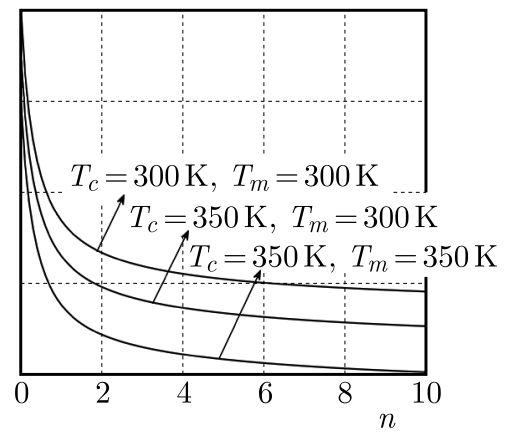

Fig. 2. Characteristic curves of the frequency-volume fraction index: (a) effect of initial condition, (b) effect of rotational speed, (c) the effect of surface temperature

Figure 3 presents characteristic curves of the natural frequency varying with rotational speed, which indicates that the natural frequency increases with rotational speed and the increasing rate becomes larger. Figure $3 \mathrm{a}\left(n=1, h=0.006 \mathrm{~m}, T_{c}=350 \mathrm{~K}\right.$ and $\left.T_{m}=300 \mathrm{~K}\right)$ shows that the natural frequency increases as the initial deflection increases. Figure $3 \mathrm{~b}\left(a_{0}=0.006 \mathrm{~m}\right.$, $h=0.006 \mathrm{~m}, T_{c}=350 \mathrm{~K}$ and $T_{m}=300 \mathrm{~K}$ ) depicts that the increasing $n$ reduces the natural frequency. Figure $3 \mathrm{c}\left(a_{0}=0.006 \mathrm{~m}, n=1, h=0.006 \mathrm{~m}\right)$ shows that the natural frequency reduces with an increase in surface temperature.

(a)

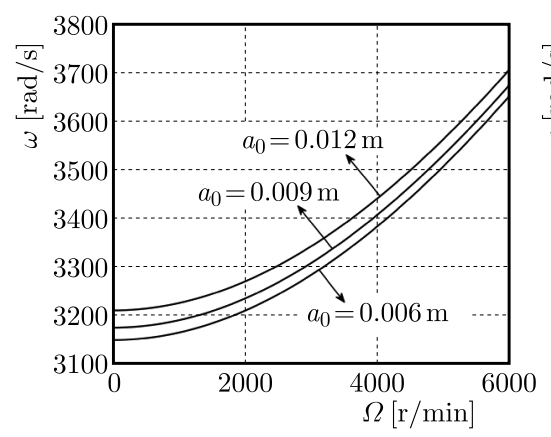

(b)

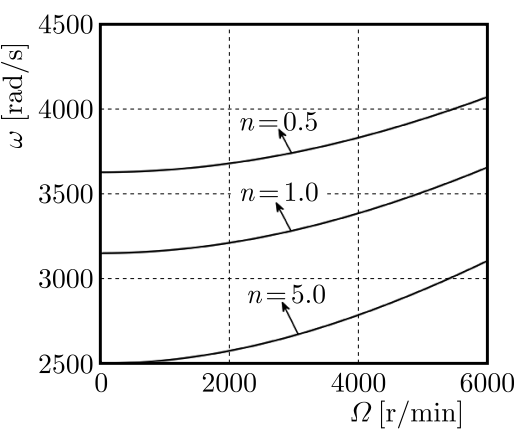

(c)

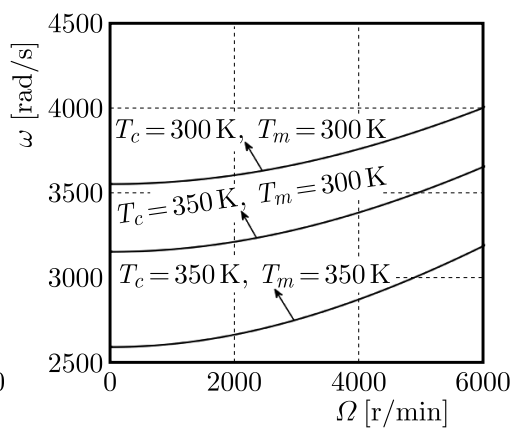

Fig. 3. Characteristic curves of frequency-rotational speed: (a) effect of initial condition, (b) effect of volume fraction index, (c) effect of surface temperature

Figure 4 presents characteristic curves of the natural frequency varying with temperature when only temperature of the ceramic side changes, but the metal surface temperature $T_{m}=T_{0}$ is kept constant. It is noted that when the ceramic surface temperature $T_{c}$ increases, because the thermal film force term $N_{T}$ is negative in the stiffness term $d_{2}$, the thermal internal force and coefficient of stiffness decrease, hence, the natural frequency decreases. The curves in Fig. 4a $(n=1, \Omega=3000 \mathrm{r} / \mathrm{min}$ and $h=0.006 \mathrm{~m})$, Fig. $4 \mathrm{~b}\left(a_{0}=0.006 \mathrm{~m}, n=1\right.$ and $\left.h=0.006 \mathrm{~m}\right)$ and Fig. $4 \mathrm{c}\left(a_{0}=0.006 \mathrm{~m}, \Omega=3000 \mathrm{r} / \mathrm{min}\right.$ and $\left.h=0.006 \mathrm{~m}\right)$ show that when temperature 
of the ceramic surface increases, the natural frequency decreases with a decrease in the initial amplitude or rotational speed as well as with an increase of $n$. This is because growth of $n$ may increase the metal content and increase the average density. Decreasing the rotational speed reduces the coefficient of stiffness.

(a)

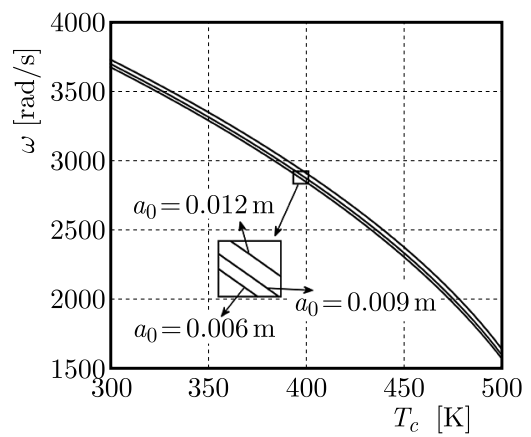

(b)

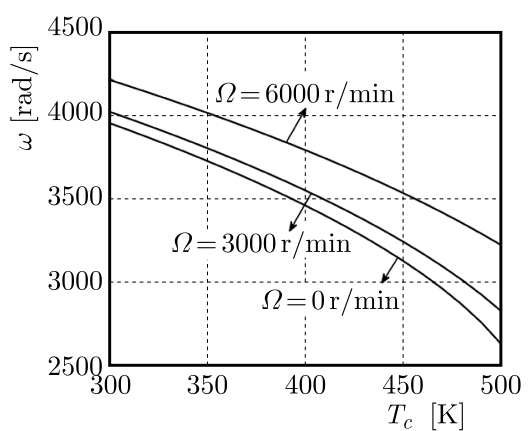

(c)

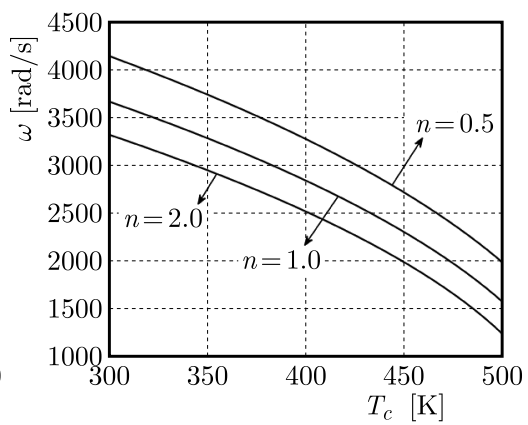

Fig. 4. Frequency-ceramic surface temperature characteristic curves: (a) effect of initial condition, (b) effect of rotational speed, (c) effect of volume fraction index

\subsection{Law of variation of static deflection with different parameters}

Figure 5 displays characteristic curves of static deflection of the rotating FGM ring plate with different parameters. As shown in Fig. $5 \mathrm{a}\left(a_{0}=0.006 \mathrm{~m}, h=0.006 \mathrm{~m}, T_{c}=350 \mathrm{~K}\right.$ and $\left.T_{m}=300 \mathrm{~K}\right)$, static deflection is caused by rotational speed. When rotational speed is zero, the static deflection is always zero even when the volume fraction index $n$ increases. However, when rotational speed is not zero and $n$ increases, the static deflection grows first and then decreases. This is because of the power relationship between the physical property parameters of the FGM plate and the volume fraction index, and there is a maximum static deflection near $n=2$. According to Fig. $5 \mathrm{~b}\left(a_{0}=0.006 \mathrm{~m}, h=0.006 \mathrm{~m}, T_{c}=350 \mathrm{~K}\right.$ and $\left.T_{m}=300 \mathrm{~K}\right)$, it can be concluded that the static deflection increases with an increase in rotational speed, but when $n$ is zero, the static deflection is a fixed value, which is because the plate degenerates into various directions of an isotropic plate, and there is no uneven distribution for density of the plate in the direction of $z$. Similarly, from Fig. $5 \mathrm{c}\left(a_{0}=0.006 \mathrm{~m}, \Omega=3000 \mathrm{r} / \mathrm{min}, h=0.006 \mathrm{~m}\right.$ and $T_{m}=300 \mathrm{~K}$ ), it can be seen that the static deflection increases with an increase in temperature on the ceramic side surface. This is because the increase of surface temperature results in growth of the thermal film force, which increases the static deflection. However, when the volume fraction index is zero, the plate degenerates into an isotropic plate and there is no static deflection.

(a)

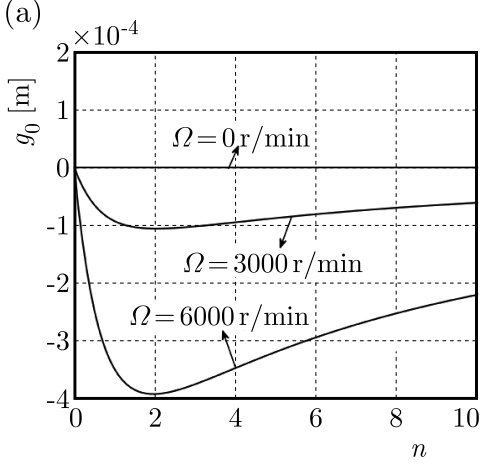

(b)

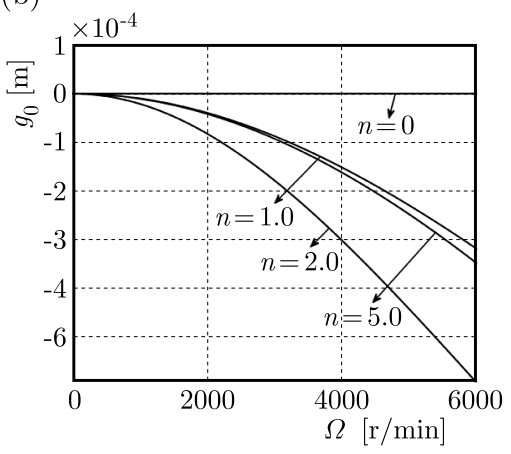

(c)

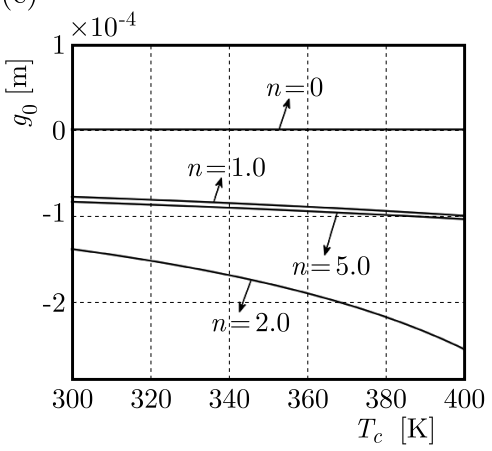

Fig. 5. Characteristic curves of static deflection as a function of different parameters: (a) variation with $n$, (b) variation with $\Omega$, (c) variation with $T_{c}$ 
Figure 6 presents the phase diagram between the displacement and speed of the FGM ring plate. Figure $6 \mathrm{a}\left(a_{0}=0.006 \mathrm{~m}, \Omega=3000 \mathrm{r} / \mathrm{min}, h=0.006 \mathrm{~m}, T_{c}=350 \mathrm{~K}\right.$ and $\left.T_{m}=300 \mathrm{~K}\right)$ indicates that the singularity is in the center, and the rotational speed of the ring plate at the equilibrium position $\left(x^{\prime}=0, v^{\prime}=0\right)$ decreases with an increase in the volume fraction index. From Fig. $6 \mathrm{~b}\left(a_{0}=0.006 \mathrm{~m}, n=1, h=0.006 \mathrm{~m}, T_{c}=350 \mathrm{~K}\right.$ and $\left.T_{m}=300 \mathrm{~K}\right)$, we note that the rotational speed has an effect on the equilibrium position and the phase diagram. An increase in the rotational speed moves the vibration equilibrium position to the left. As the rotational speed increases, the static deflection term becomes larger. Then when the rotation speed increases from $\Omega=0 \mathrm{r} / \mathrm{min}$ to $\Omega=3000 \mathrm{r} / \mathrm{min}$ and $\Omega=6000 \mathrm{r} / \mathrm{min}$, accordingly, the equilibrium position may change from $x=0 \mathrm{~m}$ to $x=-0.000015 \mathrm{~m}$ and $x=-0.0003 \mathrm{~m}$, and the speed at the equilibrium position may also increase. Figure $6 \mathrm{c}\left(a_{0}=0.006 \mathrm{~m}, n=1, \Omega=3000 \mathrm{r} / \mathrm{min}, h=0.006 \mathrm{~m}\right.$ and $\left.T_{m}=300 \mathrm{~K}\right)$ presents that for increasing the surface temperature of ceramics only, the equilibrium position of the plate is basically the zero point, which means that the influence of the static load is very small in this situation. As temperature increases, the nonlinear natural frequency of the plate decreases, resulting in a decrease in the rotational speed of the equilibrium position $\left(x^{\prime}=0, v^{\prime}=0\right)$.

(a)

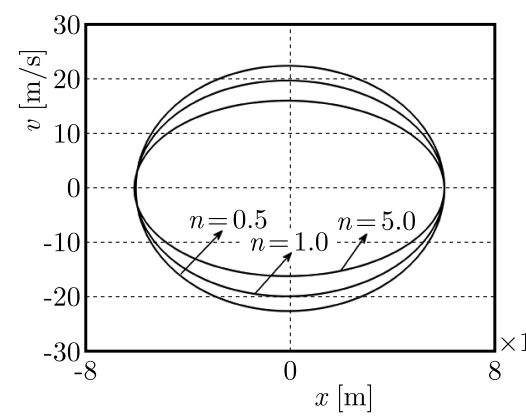

(b)

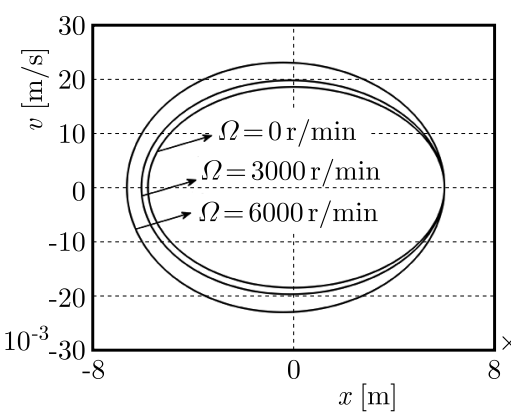

(c)

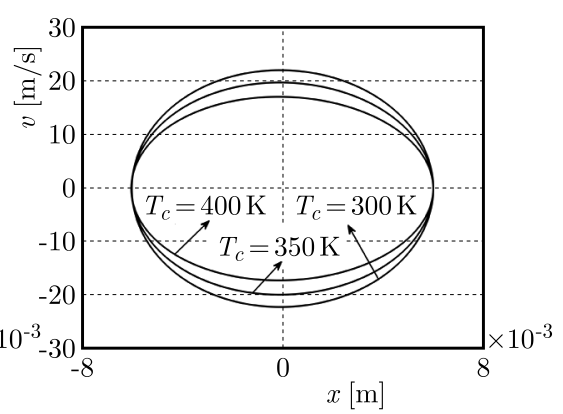

Fig. 6. The phase diagram: (a) the effect of $n$, (b) the effect of $\Omega$, (c) the effect of $T_{c}$

\subsection{Comparison of calculation results}

Figure 7 shows a comparison between the analytical solution obtained by the L-P method and the numerical solution obtained by the Runge-Kutta method for the nonlinear free vibration response of the FGM ring plate. In Fig. 7 , the parameters are: $a_{0}=0.006 \mathrm{~m}, n=1$, $\Omega=3000 \mathrm{r} / \mathrm{min}, h=0.006 \mathrm{~m}, T_{c}=350 \mathrm{~K}$ and $T_{m}=300 \mathrm{~K}$. The results show that the analytical results agree well with the numerical ones.

(a)

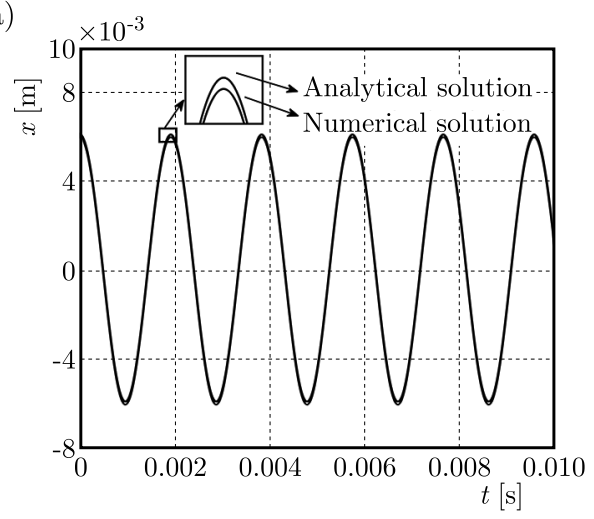

(b)

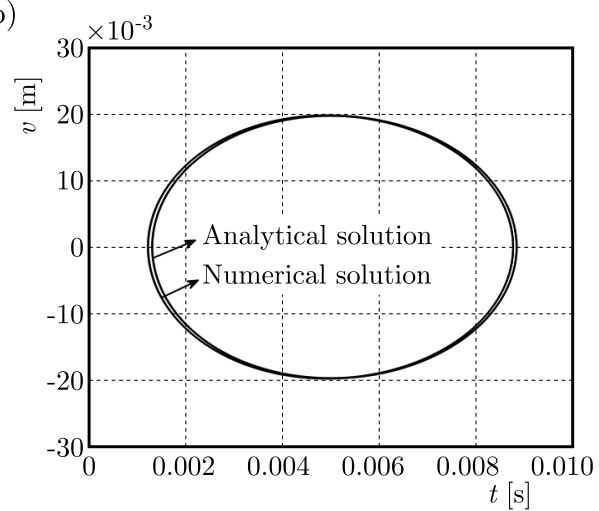

Fig. 7. Comparison diagrams: (a) response diagram, (b) comparison of phase diagram 
Figure 8 presents the power spectrum of the FGM rotating plate. The parameters in Fig. 8 are the same as those in Fig. 6. Three points are respectively taken in Fig. 2a, Fig. 3a and Fig. 4a, that is, when the initial value is $a_{0}=0.006 \mathrm{~m}$, three different volume fraction indexes $(n=0.5$, $n=1, n=5)$, rotational speeds $(\Omega=0 \mathrm{r} / \mathrm{min}, \Omega=3000 \mathrm{r} / \mathrm{min}, \Omega=6000 \mathrm{r} / \mathrm{min})$ and surface temperatures $\left(T_{c}=300 \mathrm{~K}, T_{c}=350 \mathrm{~K}, T_{c}=400 \mathrm{~K}\right)$. In Fig. 8, the natural frequency from the analytical solution can be obtained, and then numerical calculation is performed by the Runge-Kutta and Fourier transform method according to equation (3.6). Then the power spectrum diagrams are given in Fig. 8 respectively. Table 2 shows the comparison results between the specific analytical and numerical solutions. The results are basically consistent, which further illustrates reliability of the analytical solution in this paper.
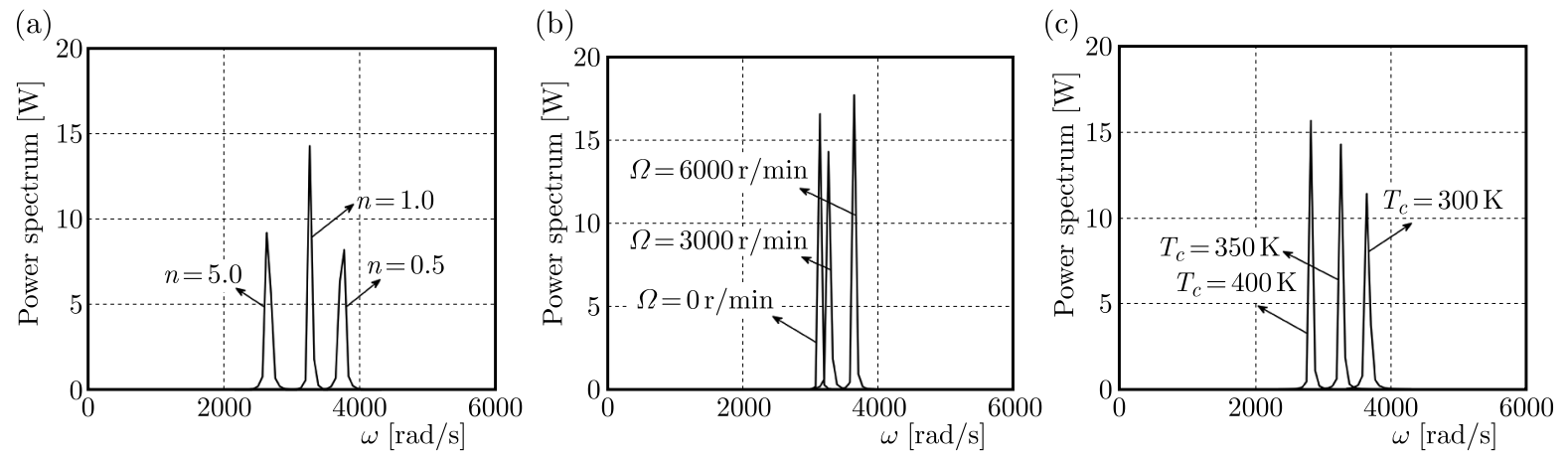

Fig. 8. The power spectrum of different parameters: (a) power spectrum for different $n$, (b) power spectrum for different $\Omega$, (c) power spectrum for different $T_{c}$

Table 2. Comparison of analytical and numerical solutions for natural frequencies

\begin{tabular}{|c|c|c|c|}
\hline $\begin{array}{c}\text { Figure } \\
\text { number }\end{array}$ & $\begin{array}{c}\text { Different } \\
\text { parameters }\end{array}$ & $\begin{array}{c}\text { Analytical } \\
\text { solution }\end{array}$ & $\begin{array}{c}\text { Numerical } \\
\text { solution }\end{array}$ \\
\hline \hline \multirow{3}{*}{ Fig. 2} & $n=0.5$ & $3740.3 \mathrm{rad} / \mathrm{s}$ & $3768 \mathrm{rad} / \mathrm{s}$ \\
\cline { 2 - 4 } & $n=1$ & $3283.9 \mathrm{rad} / \mathrm{s}$ & $3266 \mathrm{rad} / \mathrm{s}$ \\
\cline { 2 - 4 } & $n=5$ & $2666.2 \mathrm{rad} / \mathrm{s}$ & $2638 \mathrm{rad} / \mathrm{s}$ \\
\hline \multirow{3}{*}{ Fig. 3 } & $\Omega=0 \mathrm{r} / \mathrm{min}$ & $3149.9 \mathrm{rad} / \mathrm{s}$ & $3140 \mathrm{rad} / \mathrm{s}$ \\
\cline { 2 - 4 } & $\Omega=3000 \mathrm{r} / \mathrm{min}$ & $3283.9 \mathrm{rad} / \mathrm{s}$ & $3266 \mathrm{rad} / \mathrm{s}$ \\
\cline { 2 - 4 } & $\Omega=6000 \mathrm{r} / \mathrm{min}$ & $3654.2 \mathrm{rad} / \mathrm{s}$ & $3642 \mathrm{rad} / \mathrm{s}$ \\
\hline \multirow{3}{*}{ Fig. 4 } & $T_{c}=300 \mathrm{~K}$ & $3667.0 \mathrm{rad} / \mathrm{s}$ & $3642 \mathrm{rad} / \mathrm{s}$ \\
\cline { 2 - 4 } & $T_{c}=350 \mathrm{~K}$ & $3283.9 \mathrm{rad} / \mathrm{s}$ & $3266 \mathrm{rad} / \mathrm{s}$ \\
\cline { 2 - 5 } & $T_{c}=400 \mathrm{~K}$ & $2840.4 \mathrm{rad} / \mathrm{s}$ & $2826 \mathrm{rad} / \mathrm{s}$ \\
\hline
\end{tabular}

\section{Conclusions}

In this paper, based on the nonlinear elastic theory and improved L-P method, strongly nonlinear natural vibration of an FGM ring plate is studied and an analytical solution and expression for the natural frequency of the strongly nonlinear system are obtained. The results can be summarized as follows:

- With an increase in the rotational speed, the natural frequency of transverse nonlinear vibration of the FGM plate increases. Moreover, the rotational speed causes that the static load may lead to an amplitude shift. 
- Compared with the linear system, the nonlinear natural vibration is affected by the initial amplitude, where the natural frequency increases with an increase of the initial amplitude, however, decreases with an increase of surface temperature of the plate.

- In thermal environment, the natural frequency firstly increases and then decreases with growth of the volume fraction index. The static deflection increases with an increase of the rotational speed and surface temperature.

\section{Acknowledgment}

This work is supported by the National Natural Science Foundation of China (No. 11472239) and Hebei Provincial Natural Science Foundation of China (No. A2020203007).

\section{References}

1. Chen Y.R., Chen L.W., 2007, Vibration and stability of rotating polar orthotropic sandwich annular plates with a viscoelastic core layer, Composite Structures, 78, 1, 45-57

2. Chonan S., Mikami T., Ishikawa H., 1986, The vibrations and critical speeds of rotating sawblades, Transactions of the Japan Society of Mechanical Engineers, 52, 478, 1805-1812

3. Dai T., DAi H.L., 2016, Thermo-elastic analysis of a functionally graded rotating hollow circular disk with variable thickness and angular speed, Applied Mathematical Modelling, 40, 7-18, 76897707

4. Hashemi S.H., Farhadi S., Carra S., 2009, Free vibration analysis of rotating thick plates, Journal of Sound and Vibration, 323, 1-2, 366-384

5. Hu Y.D., PiaO J.M., Li W.Q., 2017, Magneto-elastic dynamics and bifurcation of rotating annular plate, Chinese Physics B, 26, 9, 094302

6. Hu Y.D., Zhang Z.Z., 2012, The bifurcation analysis on the circular functionally graded plate with combination resonances, Nonlinear Dynamics, 67, 3, 1779-1790

7. Lamb H., Southwell R.V., 1921, The vibration of spinning disk, Proceedings of the Royal Society, 99, 272-280

8. Li L.F., Wang X.Z., Zhou Y.H., 2011, Dynamic characteristics of traveling waves for a rotating laminated circular plate with viscoelastic core layer, Journal of Sound and Vibration, 330, 12, 2836-2847

9. MA L.S., WANG T.J., 2003, Nonlinear bending and post-buckling of a functionally graded circular plate under mechanical and thermal loadings, International Journal of Solids and Structures, 40, $13-14,3311-3330$

10. MA L.S., WANG T.J., 2004, Relationships between axisymmetric bending and buckling solutions of FGM circular plates based on third-order plate theory and classical plate theory, International Journal of Solids and Structures, 41, 1, 85-101

11. Malekzadeh P., Golbahar Haghighi M.R., Atashi M.M., 2011, Free vibration analysis of elastically supported functionally graded annular plates subjected to thermal environment, Acta Mechanica, 46, 7, 893-913

12. Norouzi H., Younesian D., 2015, Forced vibration analysis of spinning disks subjected to transverse loads, International Journal of Structural Stability and Dynamics, 15, 3, 1-18

13. Nosier A., Fallah F., 2009, Non-linear analysis of functionally graded circular plates under asymmetric transverse loading, International Journal of Non-Linear Mechanics, 44, 8, 928-942

14. Reddy J.N., Chin C.D., 1998, Thermomechanical analysis of functionally graded cylinders and plates, Journal of Thermal Stress, 21, 6, 593-629

15. Shakouri M., 2019, Free vibration analysis of functionally graded rotating conical shells in thermal environment, Composites Part B: Engineering, 163, 15, 574-584 
16. SHEN H.S., 2007, Thermal postbulking behavior of shear deformable FGM plates with temperature-dependent properties, International Journal of Mechanical Sciences, 49, 4, 466-478

17. Southwell R.V., 1992, On the free transverse vibrations of a uniform circular disc clamped at its centre and on the effects of rotation, Proceedings of the Royal Society A, 101, 709, 133-153

18. Yousefitabar M., Matapouri M.K., 2017, Thermally induced buckling of thin annular FGM plates, Journal of the Brazilian Society of Mechanical Sciences and Engineering, 39, 3, 969-980

19. ŻUR K.K., 2018, Free vibration analysis of elastically supported functionally graded annular plates via quasi-Green's function method, Composites Part B: Engineering, 144, 1, 37-55

Manuscript received June 28, 2020; accepted for print November 2, 2020 